\title{
Reliability and Clinical Relevance of Segmental Analysis Based on Intelligibility Assessment
}

\author{
Gwen Van Nuffelen ${ }^{\mathrm{a}}$ Marc De Bodt ${ }^{\mathrm{b}}$ Cindy Guns ${ }^{\mathrm{a}}$ Floris Wuyts ${ }^{\mathrm{a}}$ \\ Paul Van de Heyning ${ }^{\text {a }}$ \\ Antwerp University Hospital, Universities of ${ }^{\mathrm{a}}$ Antwerp and ${ }^{\mathrm{b}}$ Ghent, Belgium
}

\section{Key Words}

Segmental analysis $\cdot$ Speech $\cdot$ Reliability $\cdot$ Intelligibility

\begin{abstract}
Objective: In persons with a speech pathology, improved intelligibility is the fundamental goal of treatment. Identification of specific phonetic features that affect intelligibility, contributes to selecting intervention targets. The main purpose of this study was to investigate whether a segmental analysis based on a perceptual phoneme intelligibility assessment is reliable and can be used for target selection. Secondly, the hypothesis that the degree of agreement for phoneme identification may depend on the intelligibility of the speaker is addressed. Patients and Methods: 30 patients with pathological speech (11 dysarthric, 10 hearing impaired, 9 laryngectomees) were assessed by means of a standardized phoneme intelligibility assessment. The samples were judged twice by 9 experienced listeners. The inter- and intrarater reliability for each test item were examined. Results: The inter-rater reliability per patient for phoneme identification varied between fair and almost perfect (к: 0.24-0.89). The higher the patient's intelligibility, the higher the level of agreement $(p<0.001)$. The intra-rater reliability for phoneme identification across all patients was good ( $\mathrm{k}$ : 0.6030.787). Conclusion: A segmental analysis based on a pho-
\end{abstract}

neme intelligibility assessment is only reliable and clinically relevant in patients with a slightly to moderately decreased overall intelligibility level.

Copyright $\odot 2008$ S. Karger AG, Basel

\section{Introduction}

Increasing intelligibility is considered as the fundamental goal of therapeutic intervention and consequently intelligibility as the most important measure for speech disorders [1-3]. Intelligibility can be measured at several linguistic levels and by means of several different formats, e.g. rating scale, multiple choice, transcription, and so forth. However, intelligibility assessments are generally designed to provide the clinician an overall index of severity and an objective measure of influence of the speech production deficit on a listener $[3,4]$. Intelligibility tests also make it possible to quantify changes in speech production skills secondary to disease progression, spontaneous recovery and/or intervention. Although overall intelligibility measures do provide relevant and important information, they do not allow selection of intervention targets $[3,4]$. To develop a suitable management strategy, the intelligibility measure should be supplemented with an overview of features affecting

\section{KARGER}

Fax +4161306 1234

E-Mail karger@karger.ch

www.karger.com
(C) 2008 S. Karger AG, Basel

1021-7762/08/0605-0264\$24.50/0

Accessible online at:

www.karger.com/fpl
Gwen Van Nuffelen

Antwerp University Hospital, ORL Department

Bankwegelhof 81, BE-9120 Beveren (Belgium)

Tel. +32 382134 85, Fax +323821 4451

E-Mail gwen.van.nuffelen@telenet.be 
intelligibility. It has been argued that a well-constructed, standardized intelligibility assessment can be used to extract detailed information of phonetic features that account for impaired intelligibility [4]. The most widely known intelligibility tests allowing for such a segmental analysis are probably the Multiple Choice Word Intelligibility Test and the Paired Word Intelligibility Test [4] which are designed specifically for intelligibility testing in dysarthria. Other tests developed for the same purpose are the Swedish Intelligibility Test of Lillvik et al. [5], the Phoneme Identification Task [6] and the Dutch Intelligibility Assessment (DIA) [7]. Of course, detailed information about impaired phonetic features only makes a relevant contribution to management decisions if the information is reliable. Or in other words, such a segmental analysis is only clinically relevant if there is sufficient inter- and intra-rater agreement for phoneme identification, independently whether the perceived phoneme corresponds with the target phoneme or not. In case that the target phoneme $/ \mathrm{p} /$ is perceived as a $/ \mathrm{p} /$ by one clinician, as a $/ \mathrm{b} /$ by another clinician and as a $/ \mathrm{m} /$ by still another one, the intervention targets based on the segmental analysis would differ from clinician to clinician. Consequently, a considerable degree of inter- and intra-rater agreement for exact phoneme identification is needed.

Although highly important and clinically relevant, literature reveals limited information concerning the reliability of segmental analyses based on phoneme or word intelligibility assessments. A study of Bunton and Weismer [8] revealed information about the reliability of error rates for each of the 19 phonetic contrasts investigated by the Multiple Choice Word Intelligibility Test [4]. Strong inter-and intra-rater agreement were found for the speech productions of 25 dysarthric speakers and 10 normal subjects. In contrast, Lillvik et al. [5] found low inter- and intra-judge agreements for the segmental analysis of 9 dysarthric speakers based on a computerized word and sentence intelligibility assessment. In addition, the agreement increased with increased intelligibility, suggesting that management targets based on a segmental analysis of an intelligibility assessment are only valuable and relevant if the speaker has a certain degree of intelligibility. However, Whitehill and Ciocca [2,9] also reported strong point-to-point reliability for the transcription of $100 \mathrm{sin}$ gle words produced by Cantonese speakers with cerebral palsy. In conclusion, the available data are limited and heterogeneous.

The purpose of the current study is to investigate whether and to which extent segmental analyses based on a standardized phoneme intelligibility assessment are re- liable. Or in other words, is there a sufficient degree of inter- and intra-rater agreement for exact phoneme identification? The hypothesis that there may be a relationship between the phoneme intelligibility of a speaker and the inter-rater agreement for phoneme identification for that speaker was also investigated.

\section{Subjects and Methods}

\section{Subjects}

30 subjects participated in this study (13 women, 17 men; $12-$ 85 years of age). The subjects included 11 persons with dysarthria associated with various aetiologies, 9 persons with laryngectomees ( 7 total, 2 partial) and 10 subjects with impaired speech secondary to hearing impairment. The speakers represented a wide range of severity. The patients all signed an informed consent, which has been approved by the Ethical Committee of the Antwerp University Hospital.

\section{Listeners}

Since error analyses of speech are usually performed by clinicians, experienced listeners were preferred above naive judges. Nine experienced judges between 23 and 58 years of age were selected. The speech-language pathologists all worked on a daily basis in a rehabilitation centre and had at least 3 years of experience with the speech pathologies included in this study. The selected clinicians had no history of hearing loss.

\section{Intelligibility Assessment}

For this study the DIA [7] was used. The DIA was constructed to measure intelligibility at phoneme level and to give the clinician an overview of segmental articulatory errors made by the speaker.

Speakers are recorded while reading a series of 50 consonantvowel-consonant words. The 50 test items are separated in 3 subtests: subtest A (19 words) assesses all initial consonants, subtest $\mathrm{B}$ (15 words) all final consonants, and subtest C (16 words) all medial vowels and diphthongs of Dutch. For each test item the judges view a word frame such as '.it' (in case that the initial consonant is the target phoneme) and are asked to identify the missing phoneme. Since the phonemes of which the listener can choose from are presented next to the frame, the DIA uses a forced choice format. However, since all Dutch phonemes that may occur in a specific word position are presented, the listener is not limited in his or her choice. The listener also has the opportunity to indicate an omission. All frames are selected so that a variety of real-words and well-pronounceable non-words are possible. Well-pronounceable non-words that follow the phonotactics of Dutch are included to limit the predictability of the test items. The non-words are evaluated by both speakers and listeners and did not cause difficulties in pronunciation or in judging. To limit the clinician's familiarity with the test items, there are 25 equivalent versions of each subtest available. As all Dutch phonemes are systematically assessed, a meaningful qualitative analysis can be performed. Since all Dutch phonemes are assessed, it may be assumed that the DIA is appropriate for different types and subtypes of pathological speech, e.g. dysarthric speech, speech of hearing-impaired persons, speech of laryngectomees, and so forth. 
Speech Sample Procedures

Speech samples were made of the DIA [7]. To avoid listeners' familiarization with the test items, the three subtests of the DIA were chosen randomly. Audio recordings were made by means of a Mini-disc (Sony, MZ-R700). For all subjects the mouth-to-microphone (Sony ECM-717) distance was determined at $300 \mathrm{~mm}$. The recordings were made in a quiet environment. By using the Mini-disc, digital samples with a high sampling frequency (44 $\mathrm{kHz}$ ) were collected. The digital samples were edited to remove extraneous sounds (e.g. remarks of the examiner), using free available audio editing software: Audacity ${ }^{\circledR}$. Each test item was saved as a separate wave file. The samples were played back by means of the software Winamp (Nullsoft), installed on a notebook (Acer Aspire 1680). As the samples were judged by all the listeners at the same time, 2 speakers (Magic) were used to present the samples at a level of 75-80 dB (SPL). The speaker-to-ear distance was approximately the same for all listeners.

\section{Listening Procedures}

All the listeners judged the speech samples of the 30 speakers in one listening session. To determine intra-rater reliability, the 30 speech samples were judged a second time by the same judges with a time interval of more than 1 month. The score sheets of the DIA were used. The judges were asked to encircle the identified phoneme. In case that no phoneme could be perceived at the position of the target phoneme, they were instructed to indicate the symbol ' $\varnothing$ '. To get familiar with the system, all judges completed a trial sample.

\section{Scoring and Analysing}

To calculate the inter-rater agreement for exact phoneme identification, a confusion matrix, including all assessed phonemes at both the vertical and horizontal axis, was constructed for each speaker (separate matrices were used for the 3 subtests). For each target phoneme the matrix indicates which phoneme was perceived by how many judges. Since each target phoneme was only produced once by each speaker and since the samples were judged by 9 judges, the sum across all possible perceived phonemes had to be 9 for each target phoneme. The highest degree of agreement for a certain target phoneme is reached if all judges identify the same phoneme. Whether the perceived phoneme is the target or not does not matter for the degree of inter-rater agreement. For example if the target phoneme is a /d/ but all judges identify a / $\mathrm{t} /$ the highest degree of agreement is achieved and this will reveal important and reliable information for selection of intervention targets.

The same procedure was used for the intra-rater agreement for phoneme identification. Maximum agreement is reached if the judge identified twice the same phoneme for a specific target phoneme, independently of whether it was the target phoneme or not.

The overall intelligibility score of the DIA is calculated as the percentage of correctly identified phonemes. However, since the relationship between inter-rater agreement and degree of intelligibility is addressed for each subtest separately, we calculated the degree of intelligibility for initial consonants (subtest A), final consonants (subtest B) and vowels/diphthongs (subtest C) as the sum of correctly identified phonemes.
Statistical Analysis

The inter- and intra-rater reliability for phoneme identification has been investigated by means of $\kappa$ statistics. To define the correlation between overall intelligibility and inter-rater reliability for phoneme identification, a statistical regression analyses (ANOVA) was used. All the analyses were performed by means of SPSS.v14.

\section{Results}

\section{Inter-Rater Reliability for Exact Phoneme Identification}

The $\kappa$ values across judges diverge considerably, depending on the patient. The $\kappa$ values range from 0.300 to 0.814 for subtest A (initial consonant), from 0.316 to 0.890 for subtest B (final consonant), and from 0.238 to 0.843 for subtest $C$ (medial vowel). In general the inter-rater reliability for phoneme identification varies between fair and almost perfect. Table 1 presents for each speaker the intelligibility score and corresponding inter-rater agreement (к) per subtest.

\section{Correlation between Intelligibility and Inter-Rater \\ Agreement for Phoneme Identification}

By means of statistical regression analyses (ANOVA), significant correlations ( $\mathrm{p}<0.001)$ between speaker's intelligibility and inter-rater agreement for exact phoneme identification $(\kappa)$ were found for the three subtests of the DIA. The higher the intelligibility score, the better the inter-rater reliability for the identified phonemes. This is illustrated by figure 1 for subtest A. Similar graphs could be obtained for subtests B and C.

\section{Intra-Rater Reliability for Exact Phoneme \\ Identification}

For the intra-rater reliability per judge across all patients, $\kappa$ values range from 0.603 to 0.743 for subtest $A$ (initial consonant), from 0.695 to 0.787 for subtest B (final consonant), and from 0.615 to 0.756 for subtest $\mathrm{C}$ (medial vowel).

\section{Discussion}

The main purpose of this paper was to investigate the reliability of a segmental analysis based on a phoneme intelligibility assessment. To be reliable and clinically relevant, a strong inter- and intra-rater agreement for exact phoneme identification is needed. In case that several judges all identify a different phoneme for a specific tar- 


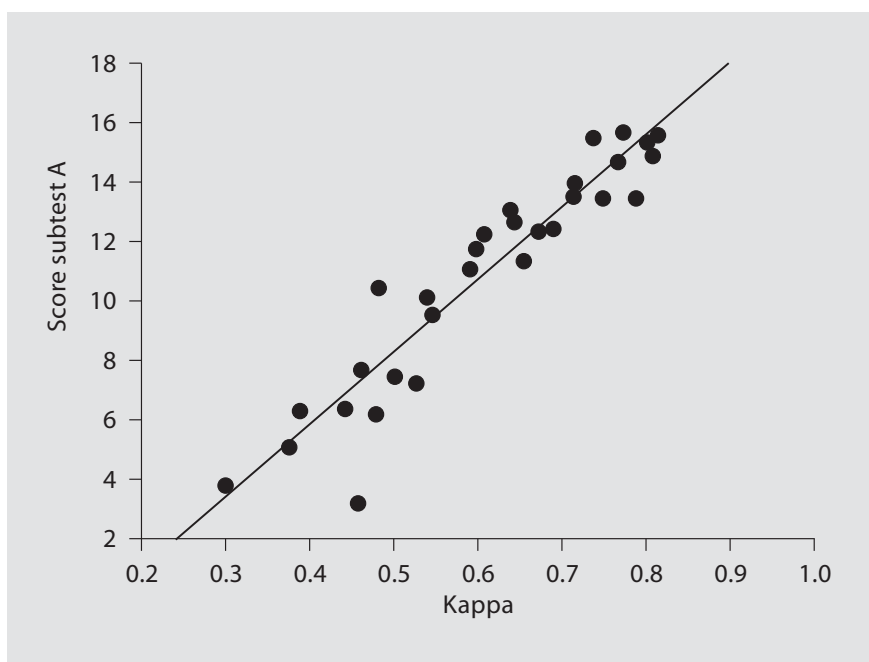

Fig. 1. Correlation per speaker between $\mathrm{k}$ and score for subtest A.

get phoneme, the analyses will not be appropriate for selecting intervention targets. However, in case that several judges do identify the same phoneme, which may differ from the target phoneme, the analysis will give the clinician valuable and reliable information for speech therapy. The results of this study reveal that the inter-rater agreement for exact phoneme identification varies among speakers depending on their phoneme intelligibility. The higher the intelligibility of a speaker, the higher the agreement for exact phoneme identification. The results consequently confirm the hypothesis that there is a relationship between the phoneme intelligibility of a speaker and the inter-rater agreement for phoneme identification. The results are also in conformity with the study of Lillvik et al. [5] who found low inter- and intra-judge agreements for the qualitative analysis of a computerized word intelligibility assessment, whereby the degree of agreement increased with increased overall intelligibility. Since the present study confirms the findings of Lillvik's group for a larger number of patients with different speech pathologies, it demonstrates that the correlation between overall intelligibility and reliability of a segmental analysis based on a well-constructed intelligibility assessment is not language- and tool-specific.

The results of this study indicate that in normal conditions (normal speaker and quiet listening environment) the human perceptual system is well able to select and interpret information from the complex acoustic signal produced by a speaker. However, decoding pathological speech seems to be a lot more difficult. Speech perception
Table 1. Intelligibility score and corresponding inter-rater agreement $(\kappa)$ per speaker and per subtest

\begin{tabular}{|c|c|c|c|c|c|c|}
\hline \multirow[t]{2}{*}{ Speaker } & \multicolumn{2}{|l|}{ Subtest A } & \multicolumn{2}{|l|}{ Subtest B } & \multicolumn{2}{|l|}{ Subtest C } \\
\hline & score $(/ 19)$ & $\kappa$ & score $(/ 15)$ & $\kappa$ & score $(/ 16)$ & к \\
\hline 1 & 13 & 0.787 & 12 & 0.736 & 9 & 0.673 \\
\hline 2 & 14 & 0.714 & 10 & 0.710 & 13 & 0.754 \\
\hline 3 & 9 & 0.460 & 8 & 0.584 & 9 & 0.692 \\
\hline 4 & 12 & 0.478 & 9 & 0.677 & 4 & 0.523 \\
\hline 5 & 15 & 0.814 & 12 & 0.824 & 11 & 0.685 \\
\hline 6 & 8 & 0.526 & 11 & 0.540 & 6 & 0.329 \\
\hline 7 & 5 & 0.442 & 7 & 0.339 & 6 & 0.418 \\
\hline 8 & 13 & 0.638 & 13 & 0.710 & 9 & 0.607 \\
\hline 9 & 16 & 0.807 & 14 & 0.734 & 12 & 0.843 \\
\hline 10 & 3 & 0.457 & 5 & 0.287 & 6 & 0.299 \\
\hline 11 & 9 & 0.539 & 8 & 0.682 & 7 & 0.652 \\
\hline 12 & 13 & 0.671 & 8 & 0.580 & 10 & 0.633 \\
\hline 13 & 13 & 0.607 & 5 & 0.429 & 9 & 0.655 \\
\hline 14 & 16 & 0.735 & 9 & 0.651 & 13 & 0.784 \\
\hline 15 & 14 & 0.642 & 14 & 0.891 & 15 & 0.890 \\
\hline 16 & 10 & 0.546 & 11 & 0.637 & 12 & 0.672 \\
\hline 17 & 3 & 0.300 & 6 & 0.600 & 2 & 0.297 \\
\hline 18 & 11 & 0.654 & 9 & 0.615 & 11 & 0.741 \\
\hline 19 & 14 & 0.747 & 13 & 0.651 & 8 & 0.715 \\
\hline 20 & 10 & 0.500 & 14 & 0.874 & 11 & 0.720 \\
\hline 21 & 13 & 0.481 & 12 & 0.749 & 11 & 0.714 \\
\hline 22 & 11 & 0.591 & 12 & 0.712 & 9 & 0.576 \\
\hline 23 & 18 & 0.773 & 13 & 0.880 & 7 & 0.486 \\
\hline 24 & 15 & 0.800 & 11 & 0.758 & 12 & 0.855 \\
\hline 25 & 14 & 0.713 & 13 & 0.730 & 11 & 0.767 \\
\hline 26 & 7 & 0.388 & 5 & 0.316 & 7 & 0.408 \\
\hline 27 & 6 & 0.375 & 9 & 0.629 & 4 & 0.238 \\
\hline 28 & 16 & 0.765 & 11 & 0.552 & 13 & 0.830 \\
\hline 29 & 13 & 0.689 & 11 & 0.850 & 7 & 0.600 \\
\hline 30 & 15 & 0.598 & 13 & 0.770 & 13 & 0.789 \\
\hline
\end{tabular}

research has shown that each listener can count on a variety of cognitive-perceptual processes and strategies to deal with variable and degraded acoustic signals [10]. It could be that in an attempt to extract as much information as possible from an impaired speech sample, different listeners use different perceptual strategies. This may result in different perceived acoustic features and/or another match between the perceived acoustic features and their mental templates for segmental events. Further research may give an answer to this assumption.

Since the phoneme intelligibility assessment, as implemented in the current experiment, does not give the listener the opportunity to indicate distortions, the current study does not provide information about the impact of distortions on the inter-rater agreement for phoneme 
identification. However, it could be expected that the occurrence and prevalence of distortions may affect interrater agreement. It would be interesting to get more insight to this process.

Based on this study it can be concluded that the reliability and consequently the clinical relevance of segmental analyses based on a phoneme intelligibility assessment, developed for that purpose, depends on speaker's intelligibility. In patients with severely decreased intelligibility, clinicians should not restrict therapy to the extracted impaired phonetic features. However, this study also shows that in case of slightly to moderately im- paired speech, the segmental analyses of a phoneme intelligibility assessment can be used for selecting targets for speech therapy.

\section{Acknowledgements}

This research was granted by the Institute for the Promotion of Innovation by Science and Technology in Flanders (IWT). The authors would like to thank their colleagues, friends and families for their assistance and also the Centre for Multiple Sclerosis of Melsbroek and the Medical Pedagogical Institute of Kwatrecht, for their cooperation.

\section{References}

1 Yorkston KM, Beukelman DR, Strand EA, Bell KR: Management of Motor Speech Disorders in Children and Adults, ed 2. Austin/ TX, Pro-ed, 1999.

-2 Whitehill TL, Ciocca V: Perceptual-phonetic predictors of single-word intelligibility: a study of Cantonese dysarthria. J Speech Lang Hear Res 2000;43:1451-1465.

- 3 Ansel BM, Kent RD: Acoustic-phonetic contrasts and intelligibility in the dysarthria associated with mixed cerebral palsy. J Speech Hear Res 1992;35:296-308.

- 4 Kent RD, Weismer G, Kent JF, Rosenbek JC: Toward phonetic intelligibility testing in dysarthria. J Speech Hear Disord 1989;54: 482-499.
5 Lillvik M, Allemark E, Karlström P, Hartelius L: Intelligibility of dysarthric speech in words and sentences: development of a computerized assessment procedure in Swedish. Logop Phoniatr Vocol 1999;24:107-119.

6 Yorkston KM, Beukelman DR, Tice R: Phoneme Identification Task: A Computer Program. Lincoln/NE, Tice Technology Services, 1998.

7 Versonnen A, De Bodt M, Van Borsel J, Wuyts FL: Een onderzoeksinstrument voor het bepalen van spraakverstaanbaarheid. Toepassing bij volwassen dysartriepatiënten [A clinical tool to determine speech intelligibility. Application to adult dysarthric speakers]. Logopedie 2004;17:33-38.
Bunton K, Weismer G: Segmental analysis of laryngeal function in persons with motor speech disorders. Folia Phoniatr Logop 2002; 54:223-239.

9 Whitehill TL, Ciocca V: Speech errors in Cantonese speaking adults with cerebral palsy. Clin Linguist Phonet 2000;14:111130.

10 Liss JM: The role of speech perception in motor speech disorders; in Weismer G (ed): Motor Speech Disorders. San Diego, Plural Publishing, 2007, pp 187-219. 